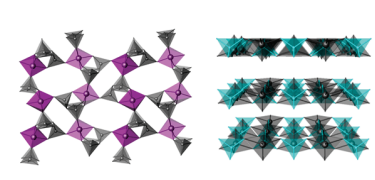

Figure 1. Structural representations of umbite (left) and natisite (right). Water molecules and charge balancing cations have been removed for clarity.

Keywords: Zirconium silicate, Titanium silicate, Ion-exchange, XAS

\section{MS14-04 Naturally occuring metal-organic frameworks}

\author{
Igor Huskić ${ }^{1}$, Tomislav Friščić ${ }^{1}$
}

1. Department of Chemistry, McGill University, 801 Sherbrooke St. West, Montreal, Canada

email: igor.huskic@mail.mcgill.ca

Metal-organic frameworks (MOFs) are a class of artificial, man-made materials, composed of organic linkers coordinated to metal ions forming networks which often exhibit increased porosity. ${ }^{[1]}$ A pinnacle of modern materials science, various MOFs have been designed and made for use in catalysis, gas storage and separation, and as molecular sensors. In our work we examine two oxalate-based minerals, Stepanovite and Zhemchuzhnikovite, which were discovered in 1960s by Knipovich and co-workers in a coal deposit near the estuary of river Lena, in the former Soviet Union. ${ }^{[2]}$ The preliminary characterisation of these materials was conducted through available methods, namely elemental analysis and powder X-ray diffraction. The latter provided crystallographic characterization of these organic minerals in terms of crystallographic symmetry and unit cell parameters. We realized that the formulas of these minerals strongly resemble those of oxalate-based MOFs first developed by Descurtins ${ }^{[3]}$ in 1990 s as novel magnetic materials. We now provide a full structural characterization of each mineral, obtained through synthetic means, which reveals two-dimensional hexagonal MOFs with honeycomb (6,3)-topology. Both minerals are made up of 2-dimensional layered networks with hexagonal cavities occupied by hexaaqua magnesium ions. While Stepanovite exhibits a rhombohedral lattice, Zhemchuzhnikovite adopts a different mode of layered stacking, leading to a hexagonal unit cell. The structures of Zhemhuznikovite and Stepanovite are, therefore, the first examples of open metal-organic frameworks found in nature. The herein presented structural analysis of these two minerals contrasts the established paradigm of MOFs as designed functional materials, and presents an unexpected new role for MOFs as naturally-ocurring minerals. The presentation will also discuss the synthetic routes through which such minerals could form, e.g. by mineral weathering of oxides recently utilized for solvent-free "accelerated aging" synthesis of microporous. ${ }^{[4]}$

[1] Kitagawa et al. Angew. Chemie 43 (2004) 2334 2375 [2] T. Echigo and M. Kimata Canadian Mineralogist 48 (2010) 1329 - 1357 [3] S. Descurtins et al. Inorg. Chem. 32 (1993) 1888-1892 [4] C. Mottillo et al. Green Chemistry 15 (2013) 2121-2131 


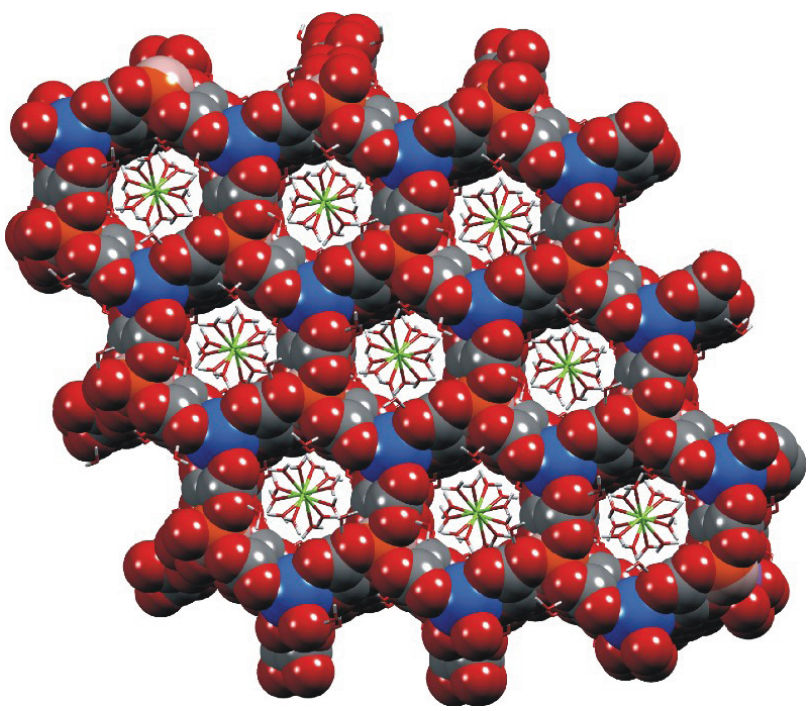

Figure 1. The structure of Zhemchuznikovite viewed down the crystallographic $c$-axis, demonstrating the overlap of neighboring sheets, which leads to the formation of channels occupied by $\mathrm{Mg}\left(\mathrm{H}_{2} \mathrm{O}\right)_{6}{ }^{2+}$ ions.

Keywords: Metal-organic frameworks, minerals, mineral weathering

\section{MS14-O5 A comprehensive analysis of the structure of imogolite nanotubes}

\author{
Stéphan ROUZIERE ${ }^{1}$, Mohamed-Salah AMARA ${ }^{1}$, Erwan \\ PAINEAU $^{1}$, Pascale LAUNOIS ${ }^{1}$
}

1. Laboratoire de Physique des Solides, UMR8502 Université Paris-Sud, Bât. 510, 91405 Orsay Cedex

email: stephan.rouziere@u-psud.fr

Imogolites, these 'tubular clays' with a diameter of the order of the nanometer, discovered in 1962 in volcanic soils [1], can now be synthesized through controllable pathways. They thus appear as most promising candidates for molecular recognition applications taking advantage of their one-dimensional porosity (catalysis, molecular sieves, sensors, etc) [2]. In 1972, natural imogolite nanotubes (INT) were proposed to have nominal composition $(\mathrm{OH})_{3} \mathrm{Al}_{2} \mathrm{O}_{3} \mathrm{SiOH}$, being formed of a curved gibbsite layer whose vacancies are bonded by isolated [Si(Ge)O $\left.\mathrm{O}_{4}\right]$ tetrahedra [3] (figure 1(a)). It was shown later that germanium can replace silicon [4]. Different studies corroborated the structural proposal of Cradwick and co-workers [3] but, to the best of our knowledge, more than forty years after their seminal article, no complete determination of INT structure has been achieved based on experimental data. We present here the first comprehensive X-ray scattering study, from small angles to wide angles, of three sorts of INT, in solution: natural and synthetic $(\mathrm{OH})_{3} \mathrm{Al}_{2} \mathrm{O}_{3} \mathrm{SiOH}$ nanotubes, with different diameters despite their similar composition, and synthetic $(\mathrm{OH})_{3} \mathrm{Al}_{2} \mathrm{O}_{3} \mathrm{GeOH}$ nanotubes [5]. We will explain the strategy we have developed to minimize interatomic distortions and to account for both small and wide angle $\mathrm{X}$-ray scattering data (figure (b)). The refined atomic structures will be discussed with respect to energetical minimizations proposed recently [6]. It is of special interest since these numerical approaches are used as guides to predict new imogolite-like structures [7]. Our methodology should also be applied in the near future to newly-discovered imogolite-type nanotubes (double-walled nanotubes or functionalized nanotubes $[2,8])$.

[1] N. Yoshinaga, S. Aomine, Soil Sci. Plant Nutr. 8, 22 (1962), [2] D.Y. Kang et al, Nat. Comm. 5, 3342 (2014); M.S. Amara et al., Chem. Mater., DOI: 10.1021/cm503428q, [3] P. Cradwick et al, Nature 240, 187 (1972), [4] S. Wada, K. Wada, Clays and Clay Minerals 30, 123 (1982), [5] M.S. Amara, S. Rouzière, E. Paineau, P. Launois, article in preparation, [6] K. Tamura, K. Kawamura, J. Phys. Chem. B 106, 271 (2002); S. Konduri et al, Phys. Rev. B 74, 033401 (2006); L. Guimarães et al, ACS Nano 1, 362 (2007); M. Zhao et al, J. Phys. Chem. C 113, 14834 (2009) ; M.P. Lourenço et al, J. Phys. Chem. C 118, 5945 (2014), [7] L. Guimarães et al, Phys. Chem. Chem. Phys. 15, 4303 (2013), [8] M.S. Amara et al, Chem. Comm. 49, 11284 (2013) 